\title{
Progress towards revealing the mechanism of herpesvirus capsid maturation and genome packaging
}

\author{
Zhihai $\mathrm{Li}^{1}$, Xuekui $\mathrm{Yu}^{1,2 \bowtie}$ \\ ${ }^{1}$ Cryo-Electron Microscopy Research Center, the CAS Key Laboratory of Receptor Research, Shanghai Institute of Materia \\ Medica, Chinese Academy of Sciences, Shanghai 201203, China \\ 2 University of Chinese Academy of Sciences, Beijing 100049, China \\ $\triangle$ Correspondence: xkyu@simm.ac.cn (X. Yu)
}

Members in the family Herpesviridae share a common architecture: the outer envelope, the inner nucleocapsid and the in-between tegument. There are three types of capsids, A-, B- and C-capsids, which are matured from the procapsid. The procapsid is assembled in the host cell nucleus where the viral dsDNA genome is packaged. The viral genome is driven into the procapsid by the terminase through the portal, which locates at a unique vertex of the icosahedral capsid (Heming et al., 2017). The A-capsid is empty and is believed to be resulted from unsuccessfully packaging of viral genome. B-capsid contains the scaffold similar to that of the procapsid and is likely resulted from failed initiation of viral DNA packaging. C-capsid contains the full-length viral genome and is able to further mature into the infectious virion. With the advance of cryo-electron microscopy (cryo-EM), the high-resolution structures of herpesvirus capsid (Yu et al., 2017; Dai and Zhou, 2018; Dai et al., 2018; Wang et al., 2018; Yuan et al., 2018) and the unique portal-vertex (Gong et al., 2019; Liu et al., 2019) of the infectious virion have been determined recently. These results showed that the structures of the herpesvirus major capsid protein and the portal, as well as the interactions among them closely resemble that of the tailed DNA bacteriophage, indicating the herpesvirus shares a common mechanism of the capsid assembly and genome package with the bacteriophage ( $\mathrm{Yu}$ et al., 2017; Dai and Zhou, 2018; Dai et al., 2018; Wang et al., 2018; Yuan et al., 2018; Gong et al., 2019; Liu et al., 2019; Chen et al., 2020). Although these achievements greatly advanced our understanding the structures and functions of herpesvirus capsid and portal, there are still questions regarding the herpesvirus capsid assembly and genome package: 1) what's the working mechanism of the herpesvirus terminase? and 2) how is the viral capsid maturation coupled with the genome packaging? Two papers published back-to-back in this issue of Protein \& Cell by Wang's group shed light on these questions (Nan Wang, 2020; Yunxiang Yang, 2020).

The researchers determined the asymmetric structures of herpesvirus simplex virus 2 (HSV-2), B-, C- and virion-capsids by using a block-based reconstruction method (Wang et al., 2018; Yuan et al., 2018; Wang et al., 2019). A comparison of asymmetric reconstructions of the portal vertex of the three capsids reveals notable differences in the position and morphology of the portal and portal vertex associated tegument (PVAT). The portal in the B-capsid locates inwards by $\sim 30 \AA$ compared to the counterparts of $\mathrm{C}$-and virioncapsids. In addition, the B-capsid portal is lack of the PVAT and is thus more accessible by the terminase, which may represent a ready-state for docking of terminase complexes. Given that the B-capsid is likely a product of unsuccessfully initiating of viral DNA package, the portal of B-capsid should somewhat resemble that of the procapsid, suggesting that the portal of procapsid could function as a DNA-packaging sensor to trigger the conformational changes of capsid during capsid maturation.

It is a long-time mystery over whether the terminase in dsDNA viruses is a pentameric rotation motor or a hexameric revolution motor (Aathavan et al., 2009; Moffitt et al., 2009; Schwartz et al., 2013; Hilbert et al., 2015; Guo et al., 2016). The first in vitro-assembled intact herpesvirus terminase complexes obtained by Wang's group are predominantly in hexamer. The researchers then determined the atomic resolution structure of the hexameric terminase complex by using block-reconstruction method. The molecular rearrangement between the ATPase domain and nuclease domain in the terminase caused by ligand binding, as well as 
the big central channel of the hexameric motor (39 $\AA$ in diameter) observed by the researchers favor the previously proposed revolution model of viral DNA translocation and cleavage. Considering the fact that a majority region in the viral portal turret (the putative site for terminase complex anchoring) in mature capsids were determined to be disordered (Gong et al., 2019; Liu et al., 2019; Nan Wang, 2020), we cannot even exclude the possibility that the DNA packaging motors have the ability to transform between pentamer and hexamer during DNA packaging. It would be fabulous to see the in situ structure of the viral terminase, while it is indeed too challenging since that tremendous efforts of many investigators over two decades failed.

\section{OPEN ACCESS}

This article is licensed under a Creative Commons Attribution 4.0 International License, which permits use, sharing, adaptation, distribution and reproduction in any medium or format, as long as you give appropriate credit to the original author(s) and the source, provide a link to the Creative Commons licence, and indicate if changes were made. The images or other third party material in this article are included in the article's Creative Commons licence, unless indicated otherwise in a credit line to the material. If material is not included in the article's Creative Commons licence and your intended use is not permitted by statutory regulation or exceeds the permitted use, you will need to obtain permission directly from the copyright holder. To view a copy of this licence, visit http:// creativecommons.org/licenses/by/4.0/.

\section{REFERENCES}

Aathavan K, Politzer AT, Kaplan A, Moffitt JR, Chemla YR, Grimes S, Jardine PJ, Anderson DL, Bustamante C (2009) Substrate interactions and promiscuity in a viral DNA packaging motor. Nature 461:669-673

Chen W, Xiao H, Wang X, Song S, Han Z, Li X, Yang F, Wang L, Song, Liu $\mathrm{H}$ et al (2020) Structural changes of a bacteriophage upon DNA packaging and maturation. Protein Cell. https://doi. org/10.1007/s13238-020-00715-9

Dai X, Zhou ZH (2018) Structure of the herpes simplex virus 1 capsid with associated tegument protein complexes. Science 360:6384

Dai X, Gong D, Lim H, Jih J, Wu TT, Sun R, Zhou ZH (2018) Structure and mutagenesis reveal essential capsid protein interactions for KSHV replication. Nature 553:521-525
Gong D, Dai X, Jih J, Liu YT, Bi GQ, Sun R, Zhou ZH (2019) DNApacking portal and capsid-associated tegument complexes in the tumor herpesvirus KSHV. Cell 178(1329-1343):e1312

Guo P, Noji H, Yengo CM, Zhao Z, Grainge I (2016) Biological nanomotors with a revolution, linear, or rotation motion mechanism. Microbiol Mol Biol Rev 80:161-186

Heming JD, Conway JF, Homa FL (2017) Herpesvirus capsid assembly and DNA packaging. Adv Anat Embryol Cell Biol 223:119-142

Hilbert BJ, Hayes JA, Stone NP, Duffy CM, Sankaran B, Kelch BA (2015) Structure and mechanism of the ATPase that powers viral genome packaging. Proc Natl Acad Sci U S A 112:E3792-3799

Liu YT, Jih J, Dai X, Bi GQ, Zhou ZH (2019) Cryo-EM structures of herpes simplex virus type 1 portal vertex and packaged genome. Nature 570:257-261

Moffitt JR, Chemla YR, Aathavan K, Grimes S, Jardine PJ, Anderson DL, Bustamante C (2009) Intersubunit coordination in a homomeric ring ATPase. Nature 457:446-450

Nan Wang WC, Zhu Ling, Feng Rui, Wang Jialing, Zhu Dongjie, Zhang Xinzheng, Liu Hongrong, Rao Zihe, Wang Xiangxi (2020) Structures of the portal vertex reveal essential protein-protein interactions for Herpesvirus assembly and maturation. Protein Cell. 16:6. https://doi.org/10.1007/s13238-020-00711-z

Schwartz C, De Donatis GM, Zhang H, Fang H, Guo P (2013) Revolution rather than rotation of $\mathrm{AAA}+$ hexameric phi29 nanomotor for viral dsDNA packaging without coiling. Virology 443:28-39

Wang J, Yuan S, Zhu D, Tang H, Wang N, Chen W, Gao Q, Li Y, Wang J, Liu $\mathrm{H}$ et al (2018) Structure of the herpes simplex virus type 2 C-capsid with capsid-vertex-specific component. Nat Commun 9:3668

Wang N, Zhao D, Wang J, Zhang Y, Wang M, Gao Y, Li F, Bu Z, Rao $Z$, Wang $X$ (2019) Architecture of African swine fever virus and implications for viral assembly. Science 366:640-644

Yu X, Jih J, Jiang J, Zhou ZH (2017) Atomic structure of the human cytomegalovirus capsid with its securing tegument layer of pp150. Science. https://doi.org/10.1126/science.aam6892

Yuan S, Wang J, Zhu D, Wang N, Gao Q, Chen W, Tang H, Wang J, Zhang X, Liu $\mathrm{H}$ et al (2018) Cryo-EM structure of a herpesvirus capsid at 3.1 A. Science. https://doi.org/10.1126/science. aao7283

Yunxiang Yang PY, Wang N, Zhu L, Zeng Y, Rao Z, Wang X (2020) Architecture of the herpesvirus genome-packaging complex and implications for DNA translocation. Protein Cell. https://doi.org/ $10.1007 /$ s $13238-020-00710-0$ 